\title{
Liposomes as a Novel Ocular Delivery System for Brinzolamide: In Vitro and In Vivo Studies
}

\author{
Huili Li, ${ }^{1}$ Yongmei Liu, ${ }^{1}$ Ying Zhang, ${ }^{2}$ Dailong Fang, ${ }^{1}$ Bei Xu, ${ }^{1}$ Lijing Zhang, ${ }^{1}$ Tong Chen, ${ }^{2}$ Ke Ren, ${ }^{3}$ Yu Nie, \\ Shaohua Yao, ${ }^{1,4}$ and Xiangrong Song ${ }^{1,4}$
}

Received 16 June 2015; accepted 28 July 2015; published online 3 September 2015

\begin{abstract}
The objective of this study was to investigate the potential of liposomes as an ophthalmic delivery system for brinzolamide (Brz) to enhance the local glaucomatous therapeutic effect. The liposomes of Brz (Brz-LPs) were produced by the thin-film dispersion method with a particle size of $84.33 \pm$ $2.02 \mathrm{~nm}$ and an entrapment efficiency of $98.32 \pm 1.61 \%$. Differential scanning calorimetry (DSC) and X-ray powder diffraction (XRD) analysis proved that Brz was successfully entrapped into Brz-LPs. Brz-LPs displayed a biphasic release pattern in vitro with burst release initially and sustained release afterwards. The corneal permeability was measured using modified Franz-type diffusion cells, and Brz-LPs showed 6.2-fold increase in the apparent permeability coefficient when compared with the commercial available formulation (Brz-Sus). Moreover, Brz-LPs $(1 \mathrm{mg} / \mathrm{mL} \mathrm{Brz})$ showed a more sustained and effective intraocular pressure reduction $(5-10 \mathrm{mmHg})$ than Brz-Sus $(10 \mathrm{mg} / \mathrm{mL} \mathrm{Brz})$ in white New Zealand rabbits. Therefore, Brz-LPs were a hopeful formulation of Brz for glaucoma treatment and worthy of further investigation.
\end{abstract}

KEY WORDS: brinzolamide; glaucoma; intraocular pressure; liposomes; permeability.

\section{INTRODUCTION}

Glaucoma is a neurodegenerative disease with high intraocular pressure (IOP) (1). According to the World Health Organization, it is the second leading cause of visual impairment and blindness in the world (2). The first-line treatment of glaucoma is topical medication to lower IOP either by decreasing the production of the aqueous humor or increasing its outflow, which could alleviate the damage of optic nerve that resulted from elevated IOP (3). The widely used eyedrops include $\alpha$ adrenergic agonists (e.g., brimonidine), beta blockers (e.g., timolol), prostaglandin analogs (e.g., latanoprost), and carbonic anhydrase inhibitors (e.g., brinzolamide).

Brinzolamide (Brz), one of the carbonic anhydrase inhibitors, is effective in reducing IOP for glaucoma therapy mainly through decreasing the production of the aqueous humor (4). Its aqueous solubility is very low at $\mathrm{pH} 7.4\left(25^{\circ} \mathrm{C}\right)$ probably due to its high lipophilicity. Therefore, the commercially

Huili Li and Yongmei Liu contributed equally to this work.

\footnotetext{
${ }^{1}$ State Key Laboratory of Biotherapy and Cancer Center, West China Hospital, Sichuan University, and Collaborative Innovation Center for Biotherapy, Chengdu, Sichuan 610041, China.

${ }^{2}$ School of Pharmaceutical Science \& Yunnan Key Laboratory of Pharmacology for Natural Products, Kunming Medical University, Kunming, Yunnan 650500, China.

${ }^{3}$ Department of Pharmaceutical Sciences, University of Nebraska Medical Center, Omaha, Nebraska 68198, USA.

${ }^{4}$ To whom correspondence should be addressed. (e-mail: shaohuayao@scu.edu.cn; songxr@scu.edu.cn)
}

available formulation of $\mathrm{Brz}(\mathrm{Brz}-\mathrm{Sus})$ is a sterile aqueous suspension (5). Brz-Sus, with obvious granular sensation, usually has poor patient compliance and low bioavailability caused by the uncomfortable feeling and subsequently undesirable tears wash to wipe the drug off rapidly after instillation. Thus, a novel local ocular delivery system for Brz needs to be developed to overcome the abovementioned problems.

Our group has successfully developed a solution formulation of Brz using the inclusion complex of hydroxypropyl- $\beta$ cyclodextrin, in which just half dosage of Brz achieved an equivalent IOP-lowing efficiency of Brz-Sus in vivo (6). We hypothesized that nanoencapsulation of Brz might further increase its local glaucomatous therapeutic efficacy and improve its patient compliance.

The aim of this work was to develop liposomes as a novel ocular delivery system for Brz (Brz-LPs) to improve its retention time and IOP-lowering efficacy. Brz-LPs were prepared by thin-film dispersion method and characterized by transmission electron microscopy, differential scanning calorimetry, X-ray diffraction, and Fourier transform infrared spectroscopy. The in vitro corneal permeability and in vivo IOP-lowering efficiency of Brz-LPs were evaluated by comparison with Brz-Sus.

\section{MATERIALS AND METHODS}

\section{Materials}

Brz (purity $>99 \%$ ) was purchased from Dalian Meilun Biology Technology Company (Dalian, China). Soybean 
phosphatidylcholine (S100) was bought from Lipoid $\mathrm{GmbH}$ (Ludwigshafen, Germany). Cholesterol was gained from Shanghai Yuanju Biology Technology Company (Shanghai, China). AZOPT ${ }^{\circledR}$ (Brz-Sus) was obtained from Alcon Laboratories (Puurs, Belgium). All other reagents were of analytical grade.

\section{Preparation of Brz-LPs}

Brz-LPs were prepared using a modified thin-film dispersion method $(7,8)$. Briefly, S100 and cholesterol were dissolved in chloroform/methanol $(4: 1, v / v)$ solvent mixture. And then, a known amount of Brz dissolved in acetone was added to the above solution. The organic solvents were subsequently removed using a rotary evaporator to produce a thin film of lipid at $37^{\circ} \mathrm{C}$. The lipid film was hydrated with $4 \mathrm{~mL}$ phosphate buffer solution at $\mathrm{pH} 7.4$ for $1 \mathrm{~h}$ at $60^{\circ} \mathrm{C}$ to obtain a suspension. Brz-LPs were finally acquired after the above suspension was sonicated for $3 \mathrm{~min}$ at $100 \mathrm{~W}$ in an ice bath and filtered through a $0.45-\mu \mathrm{m}$ syringe filter. The blank liposomes (b-LPs) were prepared following the same method without the addition of Brz.

\section{Characterization of Brz-LPs}

\section{Particle Size and Zeta Potential}

The particle size, polydispersity index, and zeta potential of Brz-LPs were determined using a Zetasizer Nano ZS90 (Malvern Instruments, Ltd., UK). The samples were suitably diluted with distilled filtered water before measurement. All measurements were carried out in triplicate.

\section{Entrapment Efficiency}

The unentrapped Brz was firstly removed from the liposomes by centrifuging at 50,000 rpm for $30 \mathrm{~min}$ at a temperature of $4{ }^{\circ} \mathrm{C}$ followed by removal of the supernatant. One milliliter of acetonitrile was then added into the liposome sediment and sonicated for $20 \mathrm{~min}$. After centrifugation at $13,000 \mathrm{rpm}$ for $10 \mathrm{~min}$, the supernatant was diluted with acetonitrile, and the content of Brz was quantified by highperformance liquid chromatography (HPLC) at $254 \mathrm{~nm}$ with a C18 column ( $4.6 \mathrm{~mm} \times 150 \mathrm{~mm} \sim 5 \mu \mathrm{m}$, Atlantis column) using acetonitrile/water $(40 / 60, v / v)$ as mobile phase. Entrapment efficiency (EE) was calculated by the following calculation equation:

$\mathrm{EE} \%=$ amount of the Brz in liposomes/initial Brz amount

$$
\times 100
$$

\section{Transmission Electron Microscopy Image}

The Brz-LPs were examined by transmission electron microscopy (TEM) (H-600, Hitachi, Japan) to characterize their morphology. A small aliquot of this suspension was stained with a drop of phosphotungstic acid (PTA, 2\%) for $30 \mathrm{~s}$ and placed on a copper grid coated with film to form a thin-film specimen. The sample was then examined and photographed with TEM at an acceleration voltage of $100 \mathrm{kV}$.

\section{DSC Analysis}

Differential scanning calorimetry (DSC) analysis was performed with a differential scanning calorimeter (TA, DSC TAQ200, USA). Around 5 6 mg samples (b-LPs, physical mixture of Brz and b-LPs (PM), pure Brz and Brz-LPs) were sealed in standard aluminum pans, respectively, and heated at a constant rate of $10^{\circ} \mathrm{C} / \mathrm{min}$ in a nitrogen atmosphere with a temperature range of $50 \sim 350^{\circ} \mathrm{C}$.

\section{XRD Analysis}

The powder samples (pure Brz, PM, b-LPs, and Brz-LPs) were examined with an X-ray power diffractometer (XRD; X'Pert Pro Philips, Netherlands) operated with $\mathrm{Cu} \mathrm{K} \alpha \mathrm{X}$ radiation, a voltage of $40 \mathrm{kV}$, and a current of $40 \mathrm{~mA}$. The scans were conducted at a scanning rate of $10 \% \mathrm{~min}$ in the $2 \theta$ range from $50^{\circ}$ to $300^{\circ}$.

\section{Fourier Transformed Infrared (FTIR) Spectra Analysis}

FTIR spectroscopy was performed using Bruker Vector 22 FT-IR Spectrometer (Bruker, Switzerland). Spectra of pure Brz, PM, b-LPs, and Brz-LPs were obtained using the $\mathrm{KBr}$ pellet method. Each $\mathrm{KBr}$ disk was scanned at $4 \mathrm{~mm} / \mathrm{s}$ over a wave number region of $400 \sim 4,000 \mathrm{~cm}^{-1}$.

\section{In Vitro Release}

The release rate of Brz from Brz-LPs was investigated by dynamic dialysis technique (9). In brief, $2 \mathrm{~mL}$ of Brz-LPs, Brz solution, and Brz-Sus were placed in different dialysis bags $(\mathrm{MWCO}=144,000 \mathrm{Da})$, respectively. Each dialysis bag was completely immersed in $40 \mathrm{~mL}$ of simulated tear fluid (STF) and continuously agitated in an orbital shaker maintained at $37^{\circ} \mathrm{C}$ at $100 \mathrm{rpm}$. At specific time intervals, $0.5 \mathrm{~mL}$ of the release medium was removed and replaced with $0.5 \mathrm{~mL}$ of the fresh release medium. The amount of Brz was quantified by the HPLC method as described above. All data were the mean of triplicate determinations.

\section{Corneal Permeability Study and Corneal Hydration Level}

The transcorneal permeability of Brz was investigated using the Franz diffusion chamber (10) which consisted of a donor and a receiver compartment (with a volume of 0.6 and $1.0 \mathrm{~mL}$, respectively). The diffusion chamber was maintained at a constant temperature $\left(37 \pm 0.2^{\circ} \mathrm{C}\right)$, under mixing conditions using a magnetic stirrer. The cornea together with a 2$\mathrm{mm}$ ring of sclera was excised within 20 min after the rabbits were sacrificed and stored in glutathione bicarbonate Ringer (GBR) buffer before use. The gained cornea was firstly fixed between clamped donor and receptor compartments through the sclera. After that, $1 \mathrm{~mL}$ of the GBR solution was added to the endothelial side, while $0.5 \mathrm{~mL}$ of Brz-LPs or Brz-Sus was added to the epithelial side. Next, $0.3 \mathrm{~mL}$ of samples was withdrawn at 15, 30, 60, 90, 120, 180, 240, 300, and $360 \mathrm{~min}$ and replaced with fresh GBR solution. The amount of drug permeation across the cornea was estimated by the HPLC method as described above. The experiment was done in triplicate. 
The apparent permeation coefficient $\left(P_{\mathrm{app}}, \mathrm{cm} / \mathrm{s}\right)$ of each formulation was calculated by Eq. (2):

$P_{\text {app }}=(\Delta Q / \Delta t) /\left(C_{0} \times A \times 60\right)$

where $C_{0}$ is the initial concentration of Brz in the donor chamber $(\mu \mathrm{g} / \mathrm{mL}), A$ is the corneal surface area for diffusion $\left(\mathrm{cm}^{2}\right), \Delta Q / \Delta t$ is the permeation rate $(\mu \mathrm{g} / \mathrm{min})$ of Brz across the intact cornea as calculated from the slope of the straight line relating the cumulative Brz permeated to time, and 60 represents the conversion of minutes to seconds. Eq. (3):

The steady state flux $\left(J_{\mathrm{ss}}, \mu \mathrm{g} / \mathrm{cm}^{2} \mathrm{~s}\right)$ was determined by

$J_{\mathrm{ss}}=C_{0} \times P_{\mathrm{app}}$ Eq. (4):

The corneal hydration level (HL, \%) was determined by

$\mathrm{HL} \%=\left(W_{\mathrm{w}}-W_{\mathrm{d}}\right) / W_{\mathrm{w}} \times 100$

where $W_{\mathrm{w}}$ was the wet cornea weight, and $W_{\mathrm{d}}$ was the corresponding dry cornea weight after a desiccation of $6 \mathrm{~h}$ at $100^{\circ} \mathrm{C}$.

\section{In Vivo IOP Measurement}

Ten male white New Zealand rabbits, $2.0 \sim 2.5 \mathrm{~kg}$, were used in this experiment. The rabbits were provided with free access to food and water in a temperature-controlled room $\left(20 \pm 5^{\circ} \mathrm{C}\right)$. The approval was obtained from the Institutional Animal Care and Use Committee of Sichuan University, and all procedures were performed in accordance with the Association for Research in Vision and Ophthalmology (ARVO) Statement on the Use of Animals in Ophthalmic Research.

Five rabbits were used for each formulation without any anesthesia $(n=5)$. Each formulation $(50 \mu \mathrm{L})$ was instilled topically into the upper quadrant of the right eye, and the IOP was measured at $0.5,1,2,3,4,5,6,8,10,12$, and $24 \mathrm{~h}$ using Tono-Pen XL® tonometer (Reichert Technologies, NY, USA) after drug administration. IOP was also measured in the undosed left eye at all mentioned time intervals according to literature $(11,12)$.

\section{Statistical Analysis}

The data were expressed as mean \pm SD. Statistical significance was assessed with paired Student's $t$ test at the 5\% level using SPSS 16.0 (SPSS Inc., Chicago, USA).

\section{RESULTS AND DISCUSSION}

\section{Preparation of Brz-LPs}

Brz-LPs were successfully prepared (7), and the process parameters were systemically optimized according to our preliminary screening study, including the lipid-to-cholesterol $(L /$ $C)$ molar ratio, lipid-to-drug $(L / D)$ molar ratio, and ultrasonic power. The detailed design for optimizing the process parameters of Brz-LPs was listed in Table I, and the results were shown in Fig. 1a-c.

As shown in Fig. 1a, the EE of Brz first increased and then reached a plateau with the increase of cholesterol molar percent. The highest EE (about 100\%) was achieved when $36 \%$ molar ratio of cholesterol (namely the $L / C$ molar ratio was 7:4) was introduced into liposomes. It is well known that cholesterol can enhance the ordered arrangement of lipid membrane and reduce the flexibility of membrane in the preparation process $(8,13,14)$. A large amount of cholesterol might hinder the penetration of Brz into the lipid bilayer, which contributed significantly to the EE improvement. However, excessive cholesterol would compete with Brz on the hydrophobic space of liposomes. Thus, further increase in the cholesterol content (when the $L / C$ molar ratio was set at 7:7) did not correspondingly enhance the Brz entrapment into liposomes.

When the $L / D$ molar ratio increased, EE first increased dramatically and then reached a plateau (Fig. 1b). Brz would precipitate during the ultrasonication and/or storage when the molar ratio of $L / D$ was $1: 1$, thereby only $65 \%$ of $E E$ was achieved. This phenomenon implied that the $L / D$ molar ratio needed to be set at higher than 1:1 in order to provide Brz with enough space of entrapment, which was similar to the literature (15). When the $L / D$ molar ratio increased to 5:1 and 10:1, EE increased significantly up to $94 \%$ and $98 \%$, respectively. However, with a further increase of the $L / D$ molar ratio to $20: 1$, the lipid film cannot be completely hydrated. Thus, the particle size markedly increased due to the high viscosity.

Figure 1c showed that the mean diameter first decreased and then increased with the increase in the ultrasonic power. The higher ultrasonic power $(100 \mathrm{~W})$ led to smaller nanodroplets which were directly related to the final size of liposomes (16). Further increase of the ultrasonic power (up to $300 \mathrm{~W}$ ) would rupture the liposomes due to the too high net shear stress, so the particle size increased and EE decreased.

According to the results described above, the molar ratio of $L / C$ was fixed to 7:4 considering a small amount of cholesterol for Brz-LP development and the smaller particle size of Brz-LP preparation. In addition, the optimal $L / D$ molar ratio was 10:1, and the best ultrasonic power was $100 \mathrm{~W}$ for Brz-LP preparation.

\section{Characterization of Brz-LPs}

\section{EE, Particle Size, and Zeta Potential}

The optimal Brz-LPs achieved an EE of $98.32 \pm 1.61 \%$ $(n=3)$. The conventional liposomes are simple vesicles consisting of outer bilayer of lipids enclosing a central aqueous core. BRZ with hydrophobic property might be entrapped in the lipid membrane; therefore, such a high EE was obtained. Brz-LPs exhibited a mean diameter of $84.33 \pm 2.02 \mathrm{~nm}(n=3)$ (Fig. 2a) with a narrow size distribution (the polydispersity index was $0.235 \pm 0.007, n=3$ ). The zeta potential of Brz-LPs was slightly negative, with the value of $-0.92 \pm 1.25 \mathrm{mV}(n=3)$. Three batches of Brz-LPs had no significant difference in EE, size, and zeta potential, which demonstrated that the preparation process was reproducible. Even though the absolute value of zeta potential was so small, our preliminary stability study had proved that Brz-LPs were physically stable for at least 
Table I. The Detailed Design for Optimizing the Process Parameters of Brz-LPs

\begin{tabular}{|c|c|c|c|c|}
\hline Optimization parameters & Variables & $\begin{array}{l}\text { S100 } \\
(\mathrm{mg})\end{array}$ & $\begin{array}{l}\text { Cholesterol } \\
(\mathrm{mg})\end{array}$ & $\begin{array}{l}\text { Drug } \\
(\mathrm{mg})\end{array}$ \\
\hline \multirow[t]{3}{*}{ Lipid-to-cholesterol molar ratio } & $7: 1$ & 62.5 & 4.5 & 4 \\
\hline & $7: 4$ & 52 & 15 & 4 \\
\hline & $7: 7$ & 45 & 22 & 4 \\
\hline \multirow[t]{4}{*}{ Lipid-to-drug molar ratio } & $1: 1$ & 6.3 & 1.7 & 4 \\
\hline & $5: 1$ & 31 & 9 & 4 \\
\hline & $10: 1$ & 63 & 18 & 4 \\
\hline & $20: 1$ & 124 & 36 & 4 \\
\hline \multirow[t]{3}{*}{ Ultrasonic power } & $50 \mathrm{~W}$ & 63 & 18 & 4 \\
\hline & $100 \mathrm{~W}$ & 63 & 18 & 4 \\
\hline & $300 \mathrm{~W}$ & 63 & 18 & 4 \\
\hline
\end{tabular}

3 days at $4^{\circ} \mathrm{C}$. The further long-term stability test would be carried out next.

\section{TEM Image}

The morphology of Brz-LPs was observed by TEM. As presented in Fig. 2b, most of the particles were nearly spherical in shape with an average particle size of approximate $80 \mathrm{~nm}$, which was accordant well with the result obtained by photon correlation spectroscopy using Zetasizer Nano ZS90.

\section{DSC Analysis}

In order to describe the existing state of Brz formulated in Brz-LPs and elucidate the entrapment mechanism of Brz into liposomes, DSC analysis was performed on b-LPs, PM, Brz-LPs, and Brz. As seen in Fig. 3a, the thermogram of Brz showed a glass transition temperature at $155^{\circ} \mathrm{C}$ and an exothermic peak at $221^{\circ} \mathrm{C}$ due to $\mathrm{Brz}$ oxidative degradation. The glass transition peak of Brz remained in PM but disappeared in Brz-LPs, which indicated that Brz was not in a crystalline state in the lipid matrix (17-19).

\section{XRD Analysis}

X-ray diffraction is a proven tool to study the crystal lattice yields and arrangements $(20,21)$. Thus, it was employed to evaluate the crystallinity of Brz-LPs compared with Brz, bLPs, and PM (Fig. 3b). The X-ray diffractogram of b-LPs showed only two major peaks with the peak intensity at $2 \theta=$ $6.95^{\circ}$ and $31.85^{\circ}$. Brz displayed several sharp and narrow peaks between $5^{\circ}$ and $50^{\circ}(2 \theta)$ with a maximum peak at $2 \theta=$ $24.97^{\circ}$ in its X-ray diffractogram, indicating the crystalline nature. Although these major sharp peaks of Brz remained in PM, there was no major peak in Brz-LPs. It was clear that the peaks of Brz disappeared from the diffraction pattern of Brz-LPs, demonstrating a marked decrease in the crystallinity of Brz in Brz-LPs and confirming the results of DSC analysis.

\section{b}

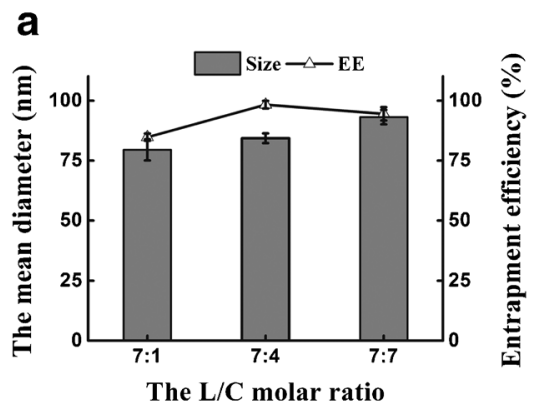

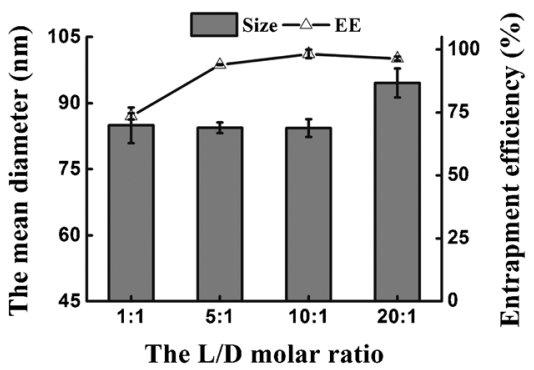

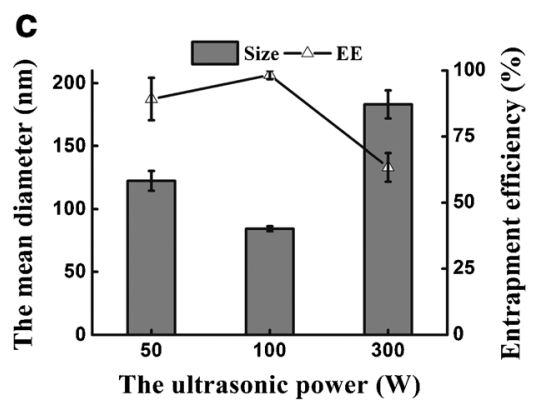

Fig. 1. Effects of various processing parameters on the mean diameter and drug entrapment efficiency of Brz-LPs, including the lipid-to-cholesterol $(L / C)$ molar ratio a, lipid-to$\operatorname{drug}(L / D)$ molar ratio b, and ultrasonic power $\mathbf{c}$. Data are expressed as mean \pm SD for $n=3$ 
a

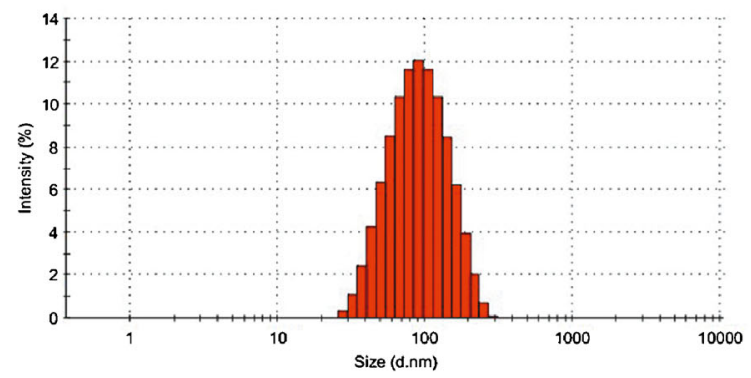

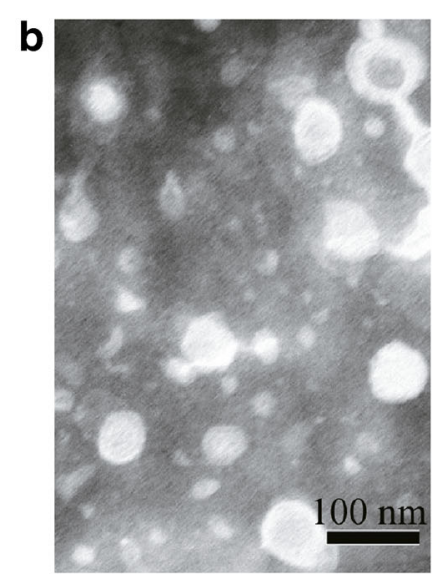

Fig. 2. The particle size distribution of Brz-LPs (a) and the transmission electron micrograph of BrzLPs. Scale bar, $100 \mathrm{~nm}(\mathbf{b})$

\section{FTIR Spectral Analysis}

FTIR spectra of Brz, b-LPs, PM, and Brz-LPs were presented in Fig. 3c. The characteristic peaks of Brz included $\mathrm{S}=\mathrm{O}$ stretch $\left(1,335.80\right.$ and $\left.1,153.35 \mathrm{~cm}^{-1}\right), \mathrm{C}-\mathrm{NH}-\mathrm{C}$ stretch $\left(3,313.89 \mathrm{~cm}^{-1}\right), \mathrm{CH}_{3}$ stretch $\left(2,962.75 \mathrm{~cm}^{-1}\right)$, and $-\mathrm{CH}_{2}$ stretch $\left(2,852.62\right.$ and $\left.1,456.18 \mathrm{~cm}^{-1}\right)$. Moreover, the absorption peaks at 651.65 and $3,097.69 \mathrm{~cm}^{-1}$ attributed to the existence of heterocycle containing $\mathrm{S}$ and $\mathrm{N}$ elements. The peaks of Brz at 3,313.89, 3,097.69, 1,335.80, 1,153.25, and $651.65 \mathrm{~cm}^{-1}$ were distinct from Brz-LPs with a clear loss in the intensity and resolution of peaks, revealing the successful incorporation of Brz. However, the characteristic absorption peaks of Brz $\left(651.89,1,139.17\right.$, and $\left.1,336.62 \mathrm{~cm}^{-1}\right)$ in PM still existed, which implied the absence of interactions between Brz and b-LPs $(22,23)$.

\section{In Vitro Release}

The in vitro release profile of Brz-LPs was illustrated in Fig. 4. A burst release (55\%) of Brz-LPs occurred in the first $1 \mathrm{~h}$. This might result from the quick release of Brz adsorbed on the surface of liposomes. Subsequently, a sustained release phase was observed in Brz-LPs within a period of $6 \mathrm{~h}(2 \sim 8 \mathrm{~h})$. After $12 \mathrm{~h}$, there almost $100 \%$ Brz was released from Brz-LPs. Compared to the release of the free drug (Brz in solution), Brz-LPs can delay the release of Brz in a way. Thus, the in vitro release study demonstrated that there was a biphasic

b

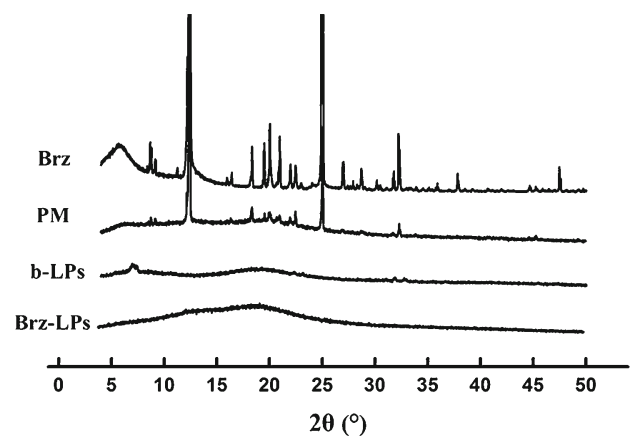

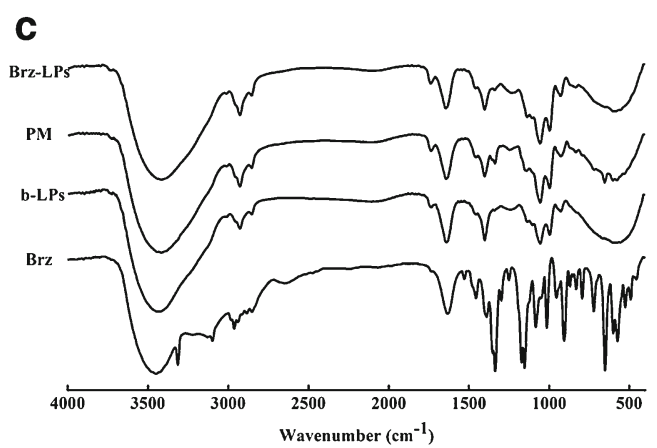

Fig. 3. Characterization of blank liposomes (b-LPs), physical mixture of Brz and b-LPs $(P M)$, Brz-loaded liposomes (Brz-LPs), and Brz (a DSC curves; b X-ray diffractograms; c FTIR spectra) 


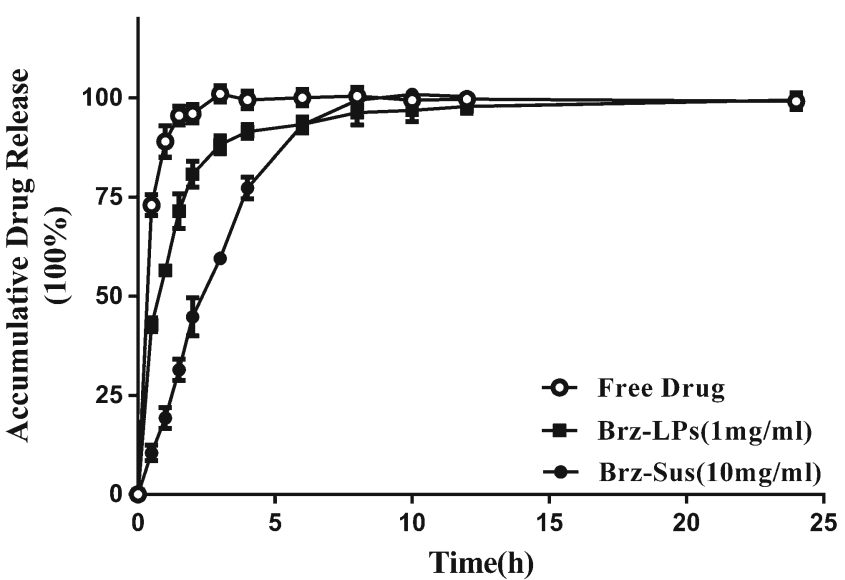

Fig. 4. In vitro release of Brz from Brz-loaded liposomes (Brz-LPs), Brz-Sus (a sterile brinzolamide aqueous suspension), and a solution of $\mathrm{Brz}$ (free drug) at $34^{\circ} \mathrm{C}$ in simulated tear fluid. Data are expressed as mean \pm SD for $n=3$

release pattern for Brz out of Brz-LPs with burst release initially and sustained release afterwards. However, Brz-Sus presented a slower sustained release compared to Brz-LPs, mainly because Brz molecules had to firstly dissolve from the large suspending particles and then diffuse through the dialysis bag.

\section{Corneal Permeability Study and Corneal Hydration Level}

Figure 5 showed the cumulative amount of $\mathrm{Brz}(\mu \mathrm{g})$ in the receptor medium over the time range $0 \sim 6 \mathrm{~h}$. A good linear relationship was obtained between the amount of Brz that had permeated through the cornea and time. Brz-LPs had no significant difference in the cumulative permeation amount at $0 \sim 3$ h compared with Brz-Sus, but a dramatic distinction appeared at $4 \sim 6 \mathrm{~h}$. The $P_{\text {app }}$ was shown in Table II. Brz-LPs showed a 6.2-fold increase in $P_{\text {app }}$ when compared with $\mathrm{Brz}$ Sus. This favorable penetration of Brz across the cornea could be attributed to the agglomeration of the liposomes as a depot

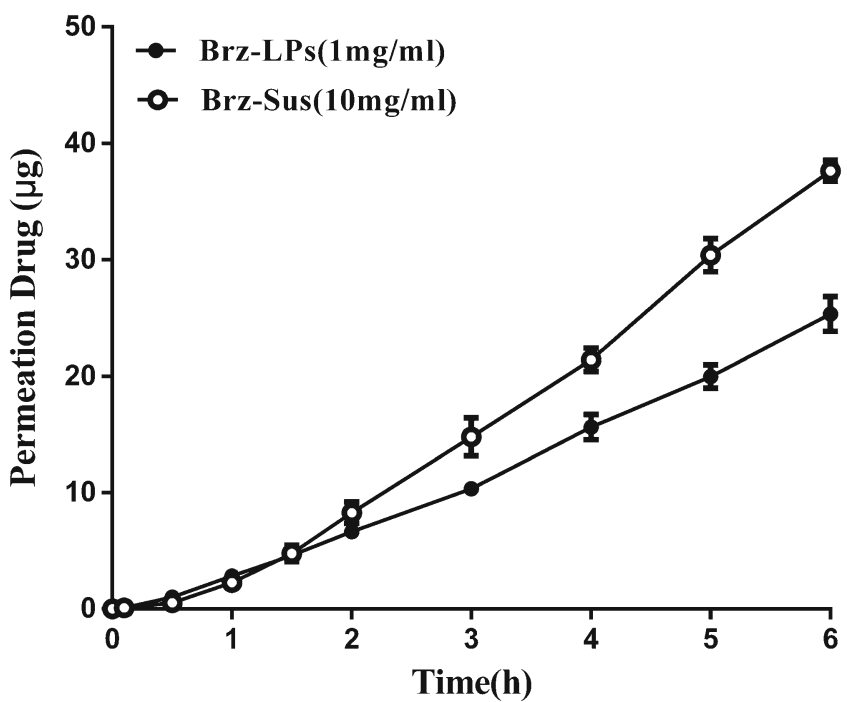

Fig. 5. The in vitro transcorneal permeation profiles of Brz in Brzloaded liposomes (Brz-LPs) and Brz-Sus (a sterile brinzolamide aqueous suspension). Data are expressed as mean \pm SD for $n=3$
Table II. Corneal Hydration Levels (HL\%) and Apparent Permeation Coefficients $\left(P_{\text {app }}\right)$ of Different Formulations Containing Brz

\begin{tabular}{lccc}
\hline Formulation & HL\% & $P_{\text {app }}\left(\times 10^{-6} \mathrm{~cm} / \mathrm{s}\right)$ & $\begin{array}{l}J_{\mathrm{ss}}\left(\times 10^{-3}\right. \\
\left.\mu \mathrm{g} / \mathrm{cm}^{2} \mathrm{~s}\right)\end{array}$ \\
\hline $1 \%$ & $80.23 \pm 2.20$ & $0.35 \pm 0.01$ & $3.54 \pm 0.08$ \\
Brz-Sus & $78.35 \pm 2.35$ & $2.58 \pm 0.04$ & $2.34 \pm 0.04$ \\
$0.1 \%$ & & & \\
Brz-LPs & & \\
\hline
\end{tabular}

Data shown are the mean \pm SD $(n=3)$

near the cornea from which the drug is slowly delivered to the precorneal area $(24,25)$.

Corneal HL is a parameter frequently used to evaluate cornea damage. Normal cornea has a HL of $75 \%$ to $80 \%$. An alteration in this level shows damage to the epithelium and/or endothelium (26). The HLs of Brz-LPs and Brz-Sus were shown in Table II. The corneal HL of Brz-LPs was $78 \%$, which indicated that Brz-LPs would not cause any damage to the corneal tissue.

\section{In Vivo IOP-Lowering Effect}

The IOP-lowering effect of Brz-LPs was examined following one topical eyedrop administration by comparison with Brz-Sus. The right eye of each animal was treated with the formulation, while its left eye was undosed for IOP comparison. After treatment, Brz-LPs $(1 \mathrm{mg} / \mathrm{mL}$ Brz $)$ presented more effective IOP reduction with a longer term role than Brz-Sus $(10 \mathrm{mg} / \mathrm{mL}$ Brz). The novel formulation of Brz had an onset in less than $30 \mathrm{~min}$, achieved a maximum IOP reduction of $41.1 \%$ at $1 \mathrm{~h}$, and sustained an effective IOP reduction until $12 \mathrm{~h}$ ( $p<0.05$ for IOP between dosed and undosed eyes), while $B r z-S u s$ began to result in an effective IOP reduction at $0.5 \mathrm{~h}$ and quickly reached its peak effect at $1 \mathrm{~h}$ (an average reduction of $34.5 \%$ from baseline as seen in Fig. 6). At $12 \mathrm{~h}$ and subsequent time points, Brz-Sus failed to effectively reduce IOP in the dosed eyes. Furthermore, the IOP was significantly

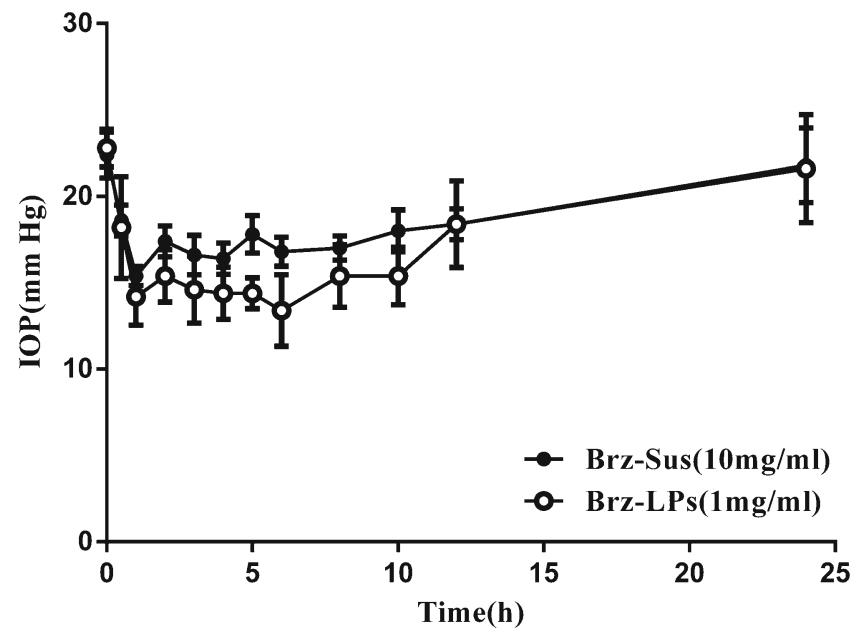

Fig. 6. The intraocular pressure $(I O P)$ reduction of white New Zealand rabbits treated by Brz-loaded liposomes $(B r z-L P s)$ and Brz-Sus (a sterile brinzolamide aqueous suspension). Data are expressed as mean \pm SD for $n=5$ 
lower in Brz-LPs group at any time point from 2 to $10 \mathrm{~h}$ than Brz-Sus group $(p<0.05)$. The higher ocular hypotensive effect produced by Brz-LPs attributed to the fact that liposomes had a higher binding affinity to the corneal surface than Brz-Sus (27), which caused better absorption of Brz. The pharmacokinetic study of Brz-LPs is in progress to further explain the mechanism of lowering IOP.

\section{CONCLUSIONS}

In this study, Brz-LPs were successfully prepared by a modified thin-film dispersion method. TEM, DSC, XRD, and FTIR results revealed the successful formation of Brz-LPs. The in vitro release of Brz from Brz-LPs was slower than free drug. Ex vivo corneal transport experiment indicated that Brz-LPs had better transmembrane permeation ability than Brz-Sus and could improve the transport of Brz across the cornea by sixfold. Brz-LPs (1 mg/mL Brz) achieved more sustained and effective reduction in IOP than Brz-Sus $(10 \mathrm{mg} / \mathrm{mL} \mathrm{Brz})$ in white New Zealand rabbits, which signified that Brz-LPs had ten times stronger efficacy in vivo than Brz-Sus. In summary, all these data supported the belief that Brz-LPs would have a hopeful future as a novel formulation of Brz for glaucoma treatment.

\section{ACKNOWLEDGMENTS}

This research has received financial support from the National Natural Science Foundation of China (No. 81302729), the Fundamental Research Funds for the Central Universities (No. 2013SCU04A19), and the Sichuan Province Science and Technology Support Program (No. 2015SZ0122).

Conflict of Interest The authors declare that they have no competing interests.

\section{REFERENCES}

1. Jung HJ, Abou-Jaoude M, Carbia BE, Plummer C, Chauhan A. Glaucoma therapy by extended release of timolol from nanoparticle loaded silicone-hydrogel contact lenses. J Control Release. 2013;165(1):82-9. doi:10.1016/j.jconrel.2012.10.010.

2. Yang H, Tyagi P, Kadam RS, Holden CA, Kompella UB. Hybrid dendrimer hydrogel/PLGA nanoparticle platform sustains drug delivery for one week and antiglaucoma effects for four days following one-time topical administration. ACS Nano. 2012;6(9):7595-606.

3. Ding L, Lee T, Wang CH. Fabrication of monodispersed taxolloaded particles using electrohydrodynamic atomization. J Control Release. 2005;102(2):395-413.

4. Siesky B, Harris A, Cantor LB, Kagemann L, Weitzman Y, McCranor L, et al. A comparative study of the effects of brinzolamide and dorzolamide on retinal oxygen saturation and ocular microcirculation in patients with primary open-angle glaucoma. Brit J Ophthalmol. 2008;92(4):500-4. doi:10.1136/ bjo.2007.125187.

5. Iester M. Brinzolamide ophthalmic suspension: a review of its pharmacology and use in the treatment of open angle glaucoma and ocular hypertension. Clin Ophthalmol. 2008;2(3):517-23.

6. Zhang Y, Ren K, He Z, Li H, Chen T, Lei Y, et al. Development of inclusion complex of brinzolamide with hydroxypropyl-betacyclodextrin. Carbohydr Polym. 2013;98(1):638-43. doi:10.1016/ j.carbpol.2013.06.052.
7. Hironaka K, Inokuchi Y, Tozuka Y, Shimazawa M, Hara H, Takeuchi H. Design and evaluation of a liposomal delivery system targeting the posterior segment of the eye. J Control Release. 2009;136(3):247-53. doi:10.1016/j.jconrel.2009.02.020.

8. Hathout RM, Mansour S, Mortada ND, Guinedi AS. Liposomes as an ocular delivery system for acetazolamide: in vitro and in vivo studies. AAPS PharmSciTech. 2007;8(1):1-12.

9. Dai Y, Zhou R, Liu L, Lu Y, Qi J, Wu W. Liposomes containing bile salts as novel ocular delivery systems for tacrolimus (FK506): in vitro characterization and improved corneal permeation. Int $\mathrm{J}$ Nanomedicine. 2013;8:1921-33. doi:10.2147/IJN.S44487.

10. Gupta H, Aqil M, Khar RK, Ali A, Bhatnagar A, Mittal G. Nanoparticles laden in situ gel for sustained ocular drug delivery. J Pharm Bioallied Sci. 2013;5(2):162-5. doi:10.4103/09757406.111824.

11. El-Gazayerly ON, Hikal AH. Preparation and evaluation of acetazolamide liposomes as an ocular delivery system. Int J Pharm Technol Res. 1997;158(2):121-7.

12. Franca JR, Foureaux G, Fuscaldi LL, Ribeiro TG, Rodrigues LB, Bravo R, et al. Bimatoprost-loaded ocular inserts as sustained release drug delivery systems for glaucoma treatment: in vitro and in vivo evaluation. PLoS One. 2014;9(4), e95461. doi:10.1371/ journal.pone.0095461.

13. Kirby C, Clarke J, Gregoriadis G. Effect of the cholesterol content of small unilamellar liposomes on their stability in vivo and in vitro. Biochem J. 1980;186(2):591-8.

14. Senior J, Gregoriadis G. Stability of small unilamellar liposomes in serum and clearance from the circulation: the effect of the phospholipid and cholesterol components. Life Sci. 1982;30(24):2123-36.

15. Zhang JA, Anyarambhatla G, Ma L, Ugwu S, Xuan T, Sardone T, et al. Development and characterization of a novel Cremophor ${ }^{\circledR}$ EL free liposome-based paclitaxel (LEP-ETU) formulation. Eur J Pharm Biopharm. 2005;59(1):177-87.

16. Barba AA, Bochicchio S, Lamberti G, Dalmoro A. Ultrasonic energy in liposome production: process modelling and size calculation. Soft Matter. 2014;10(15):2574-81. doi:10.1039/ c3sm52879k.

17. Hamoudeh M, Diab R, Fessi H, Dumontet C, Cuchet D. Paclitaxel-loaded microparticles for intratumoral administration via the TMT technique: preparation, characterization, and preliminary antitumoral evaluation. Drug Dev Ind Pharm. 2008;34(7):698-701. doi:10.1080/03639040701842444.

18. $\mathrm{Mu} \mathrm{L}$, Feng SS. Fabrication, characterization and in vitro release of paclitaxel (Taxol®) loaded poly (lactic-co-glycolic acid) microspheres prepared by spray drying technique with lipid/cholesterol emulsifiers. J Control Release. 2001;76(3):239-54.

19. Kassem MA, ElMeshad AN, Fares AR. Lyophilized sustained release mucoadhesive chitosan sponges for buccal buspirone hydrochloride delivery: formulation and in vitro evaluation. AAPS PharmSciTech. 2015;16(3):537-47. doi:10.1208/s12249-014-0243-3.

20. Dhanikula AB, Panchagnula R. Development and characterization of biodegradable chitosan films for local delivery of paclitaxel. AAPS J. 2004;6(3):88-9.

21. Dhanikula AB, Panchagnula R. Fluorescence anisotropy, FT-IR spectroscopy and 31-P NMR studies on the interaction of paclitaxel with lipid bilayers. Lipids. 2008;43(6):569-79. doi:10.1007/ s11745-008-3178-1.

22. Mladenovska K, Cruaud O, Richomme P, Belamie E, Raicki RS, Venier-Julienne MC, et al. 5-ASA loaded chitosan-Ca-alginate microparticles: preparation and physicochemical characterization. Int J Pharm Technol Res. 2007;345(1-2):59-69.

23. Verma P, Gupta RN, Jha AK, Pandey R. Development, in vitro and in vivo characterization of Eudragit RL 100 nanoparticles for improved ocular bioavailability of acetazolamide. Drug Deliv. 2013;20(7):269-76. doi:10.3109/ 10717544.2013.834417.

24. Gupta H, Aqil M, Khar RK, Ali A, Bhatnagar A, Mittal G. Biodegradable levofloxacin nanoparticles for sustained ocular drug delivery. J Drug Target. 2011;19(6):409-17. doi:10.3109/ 1061186X.2010.504268.

25. Gupta H, Aqil M, Khar RK, Ali A, Bhatnagar A, Mittal G. Sparfloxacin loaded PLGA nanoparticles for sustained ocular drug delivery. Nanomedicine. 2010;6(2):324-33. doi:10.1016/ j.nano.2009.10.004. 
26. Vega E, Gamisans F, Garcia ML, Chauvet A, Lacoulonche F, Egea MA. PLGA nanospheres for the ocular delivery of flurbiprofen: drug release and interactions. J Pharm Sci. 2008;97(12):5306-17. doi:10.1002/jps.21383.
27. Natarajan JV, Ang M, Darwitan A, Chattopadhyay S, Wong TT, Venkatraman SS. Nanomedicine for glaucoma: liposomes provide sustained release of latanoprost in the eye. Int J Nanomedicine. 2012;7:123-31. doi:10.2147/IJN.S25468. 